\title{
Impact of number of channels on RF shimming at 3T
}

\author{
Alexander S. Childs $\cdot$ Shaihan J. Malik \\ Declan P. O'Regan · Joseph V. Hajnal
}

Received: 16 July 2012/Revised: 11 December 2012/ Accepted: 14 December 2012/Published online: 13 January 2013

(C) The Author(s) 2013. This article is published with open access at Springerlink.com

\begin{abstract}
Object At high-field strengths $(\geq 3 \mathrm{~T})$ inhomogeneity of the radio frequency (RF) field and RF power deposition become increasingly problematic. Parallel Transmission (PTx) - the use of segmented transmission arrays with independently driven elements-affords the ability to combat both of these issues. There are a variety of existing designs for PTx coils, ranging from systems with two channels to systems with eight or more. In this work, we have investigated the impact of the number of independent channels on the achievable results for both homogeneity improvement and power reduction in vivo.

Materials and methods A 3T Philips Achieva MRI system fitted with an 8-channel PTx body coil was driven so as to emulate configurations with 1,24 and 8 independent channels. RF shimming was used in two different anatomies in order to assess improvements in RF homogeneity. Results Significant homogeneity improvements were observed when increasing from 1 to 2,2 to 4 , and 4 to 8 channel configurations. Reductions in RF power requirements and local SAR were predicted for increasing numbers of channels.

Conclusion Increasing the number of RF transmit channels adds extra degrees of freedom which can be used to
\end{abstract}

\section{A. S. Childs · D. P. O'Regan}

Robert Steiner MRI Unit, Imaging Sciences Department, MRC Clinical Sciences Centre, Imperial College London, Hammersmith Hospital, London, UK

\section{S. J. Malik $(\bowtie) \cdot$ J. V. Hajnal}

Division of Imaging Sciences and Biomedical Engineering, King's College London, The Rayne Institute, 4th Floor Lambeth Wing, St. Thomas' Hospital, London SE1 7EH, UK e-mail: shaihan.malik@kcl.ac.uk benefit homogeneity improvement or power reduction for body imaging at $3 \mathrm{~T}$.

Keywords Parallel Transmission · RF shimming - Image nonuniformity $\cdot$ SAR control

\section{Introduction}

At high field strengths (3T and greater), the excitation radio-frequency (RF) wavelength becomes comparable to the size of the human body, causing strong coil-tissue interactions which are subject-specific, depending on geometry and tissue electrical properties. The result of these interactions is an inhomogeneous RF transmit $\left(\mathrm{B}_{1}{ }^{+}\right)$ field, which leads to spatially varying image contrast. Furthermore, conventional single-channel RF systems generally have a one-time adjustment to match the RF coil to a nominal load presented by an "average patient", whereas in fact the loading of the RF coil is subjectdependent, which can result in variable performance. The use of multiple transmit channels offers a means for mitigating these effects by allowing for greater control over the RF electromagnetic fields. RF shimming [1], in which a subject-specific adjustment is made to the relative amplitudes and phases of each transmit channel, can be used to improve $\mathrm{B}_{1}{ }^{+}$homogeneity and/or reduce SAR.

While such techniques have been technically demonstrated for transmit arrays with eight or more channels [2, 3], systematic clinical studies of RF shimming have primarily used commercially available two channel systems [4]. RF shimming at $3 \mathrm{~T}$ has been used to stabilise and improve $\mathrm{B}_{1}{ }^{+}$homogeneity while achieving shorter image acquisition times compared with single-channel quadrature excitation [4], and to also improve diagnostic quality of 
resulting images [5]. These improvements come directly from the ability to manipulate the spatial $\mathrm{B}_{1}{ }^{+}$distribution as a linear sum of contributing fields from individual coil elements:

$S(\mathbf{r})=\sum_{i=1}^{N c} \sigma_{i}(\mathbf{r}) b_{i}$

In the above expression, $\sigma_{i}(\mathbf{r})$ is the transmit sensitivity of the $i$ th coil, $\mathrm{N}_{\mathrm{c}}$ is the total number of coils, $S(\mathbf{r})$ is the overall transmit sensitivity, and $b_{i}$ are complex weights applied to each channel. Transmit sensitivity is defined as the ratio of the actual $\mathrm{B}_{1}{ }^{+}$field to the nominal value; it is a dimensionless value which describes spatial variation of the field amplitude and phase. Equation 1 shows that the ability to obtain uniform overall sensitivity depends on the sensitivities of the different coils in the array. It might be expected, therefore, that more coils, or perhaps different coil designs, would yield even larger improvements than a two-channel configuration. In this investigation, a $3 \mathrm{~T}$ MRI scanner with eight physical transmit channels has been used to emulate different 2-, 4- and 8-channel configurations in vivo. Possible benefits of RF shimming were assessed in a multiple-volunteer study focusing on $\mathrm{B}_{1}{ }^{+}$homogeneity, image quality and RF power reduction.

\section{Methods}

Experiments were performed using an 3T Achieva MRI system (Philips Healthcare, Best, The Netherlands) equipped with an 8-channel parallel transmit (PTx) body coil. The coil [3] consists of an array of eight elements, arranged around the bore of the magnet to give maximum spatial variation in transmit sensitivity in the axial plane.

\section{RF shim framework}

The overall transmit sensitivity of the array coil is a linear sum depending on the complex weighting applied to each channel (Eq. 1). This can be rewritten in matrix form as $\mathbf{S}=\boldsymbol{\sigma b}$, where $\mathbf{S}$ is an $N \times 1$ vector ( $N$ is the number of voxels), $\boldsymbol{\sigma}$ is an $N \times N_{\mathrm{c}}$ sensitivity matrix and $\mathbf{b}$ is an $N_{\mathrm{c}} \times 1$ vector containing the complex coil weightings for each channel. For the system used, $N_{\mathrm{c}}=8$ and $\mathbf{b}=\left[\begin{array}{llllllll}1 & 1 & 1 & 1 & 1 & 1 & 1 & 1\end{array}\right.$ $1]^{\mathrm{T}}$ is defined as quadrature mode (single-channel) transmission. This definition of "quadrature" mode depends on the system being calibrated; this will be discussed later. Emulation of systems with a reduced number of channels can be performed by introducing an $N_{\mathrm{c}} \times N_{\mathrm{r}}$ channel reduction matrix, $\mathbf{R}$. This reduction matrix allows for emulation of systems with $N_{\mathrm{r}}<N_{\mathrm{c}}$ channels via linear combinations of the scanner's physical channels. The transmit sensitivity is then written $\mathbf{S}=\boldsymbol{\sigma} \mathbf{R} \mathbf{b}$, and $\mathbf{b}$ represents the weightings applied now not to individual physical coils, but to the $\mathrm{N}_{\mathrm{r}}$ emulated channels. Full control of the physical coils implies $\mathbf{R}$ is the $N_{\mathrm{c}} \times N_{\mathrm{c}}$ identity matrix.

The phase/amplitude relationship between all channels that defines quadrature mode is a property of the system hardware that must be determined by calibration. For our system, this is done using a standard phantom. Choice of the calibration conditions does not affect full 8-channel operation. However, it adds a bias to reduced channel combinations by locking in some aspects of the calibration. In order to reduce the effect that this has, a new system calibration matrix was computed by performing full 8-channel shimming on 10 previously acquired pelvic $\mathrm{B}_{1}{ }^{+}$ maps (10 different subjects) individually, then taking the complex average of all results for each channel. The resulting weights $c_{\mathrm{i}}$, which provide an optimised average starting shim, can be incorporated into a diagonal matrix, $\mathbf{C}_{\mathrm{ii}}=c_{\mathrm{i}}$ which is included in the overall expression for the combined system sensitivity: $\mathbf{S}=\boldsymbol{\sigma} \mathbf{C R b}$. The calibration matrix was explicitly defined prior to any experimental work and not altered subsequently.

RF Shim weights were calculated using Magnitude Least Squares (MLS) optimisation [6, 7] using the local variable exchange method

$\mathrm{b}=\min \left\{\left\|\left|\sigma C R b-S_{\text {target }}\right|\right\|^{2}+\lambda\|\mathrm{Rb}\|^{2}\right\}$

$\mathbf{S}_{\text {target }}$ is the target combined transmit sensitivity; in this work $\mathbf{S}_{\text {target }}=1.0$ for all space. The regularisation parameter $\lambda$ is designed to control total RF power. Note that the solution, $\mathbf{b}$, has $\mathrm{N}_{\mathrm{r}}$ entries; the actual weights applied to the $\mathrm{N}_{\mathrm{c}}$ physical coils are determined from the product $\mathbf{R b}$. Hence the second term in Eq. 2, which enumerates the total RF power, considers this product. The overall scaling of $\mathbf{R}$ is not important in the term $\lambda\|\mathbf{R b}\|^{2}$ since scalar factors in $\mathbf{R}$ can be subsumed into the parameter $\lambda$.

Several candidate reduction matrices for emulating 2- and 4-channel systems were tested on the pilot data. Candidates included phased combinations, Fourier modes [2] and pair-wise coil combinations. These were evaluated using Eq. 2 with a range of values of $\lambda$ to find the combinations that produced the optimal combination of $\mathrm{B}_{1}{ }^{+}$ homogeneity and lowest total RF power when applied to the pilot data. The optimised 4-channel reduction matrix used in this study is shown below; in this case adjacent coils were paired together, corresponding to creation of an enveloping array with fewer, larger coil elements placed adjacently.

$\mathbf{R}^{T}=\left(\begin{array}{llllllll}1 & 1 & 0 & 0 & 0 & 0 & 0 & 0 \\ 0 & 0 & 1 & 1 & 0 & 0 & 0 & 0 \\ 0 & 0 & 0 & 0 & 1 & 1 & 0 & 0 \\ 0 & 0 & 0 & 0 & 0 & 0 & 1 & 1\end{array}\right)$. 
Two 2-channel combinations were used. The first used the following reduction matrix

$\mathbf{R}^{T}=\left(\begin{array}{llllllll}1 & 1 & 0 & 0 & 1 & 1 & 0 & 0 \\ 0 & 0 & 1 & 1 & 0 & 0 & 1 & 1\end{array}\right)$

which was found to yield the best results in the pilot data. The second was chosen to emulate orthogonal, nominally linear modes of a 2-channel birdcage system and was defined as:

$\mathbf{R}^{T}=\frac{1}{2}\left(\begin{array}{cccccccc}2 & 1-i & 0 & 1+i & 2 & 1-i & 0 & 1+i \\ 0 & 1+i & 2 & 1-i & 0 & 1+i & 2 & 1-i\end{array}\right)$.

\section{RF shimming}

For each subject, experiments were performed using the three different combination matrices described above, as well as 8-channel optimization $(\mathbf{R}=\mathbf{I})$. In each case, error vs. power curves ("L-curves") were plotted by performing the optimisation (Eq. 2) for multiple values of $\lambda$. The MLS algorithm, implemented in MATLAB 2009b (The Mathworks, Natick, MA) on a desktop PC (Windows XP, Dual core CPU $2.4 \mathrm{GHz}$, 2 GB RAM), calculated all shim settings for a subject in less than $10 \mathrm{~s}$, allowing for straightforward implementation in a scanning session. The curves were used to select two solutions for each case: the first maximised the homogeneity improvement obtainable whilst using the same RF power level as quadrature mode excitation, the second minimised the RF power level while maintaining approximately the same homogeneity as quadrature excitation. Figure 1 shows how these solutions were obtained using L-curves calculated for one of the study subjects. Imaging experiments were performed for the first solutions (maximised homogeneity) where measurable differences in $\mathrm{B}_{1}{ }^{+}$were expected; in order to keep the examination duration within $1 \mathrm{~h}$ for each subject, the minimized power solutions were not used for imaging.

\section{Imaging experiments}

RF shimming experiments were carried out on healthy volunteers, with the focus being thigh and pelvis imaging. A six-element phased array coil was used for signal reception. Research ethics committee approval was obtained for the study, and all participants gave written informed consent prior to enrolment. In total, seven healthy volunteers (two male, five female) underwent pelvic and/or thigh scanning (five volunteers underwent both).

Axial $\mathrm{B}_{1}{ }^{+}$field maps were acquired using the actual flip angle imaging sequence [8] including a modified spoiling regime [9], slice profile correction [10] and linear combination mapping to improve SNR [11-13]. Imaging parameters were $\mathrm{FOV}=440 \mathrm{~mm} \times 220 \mathrm{~mm}, 64 \times 32$ matrix, slice thickness $10 \mathrm{~mm}$, nominal flip angle $=80^{\circ}$ and pulse

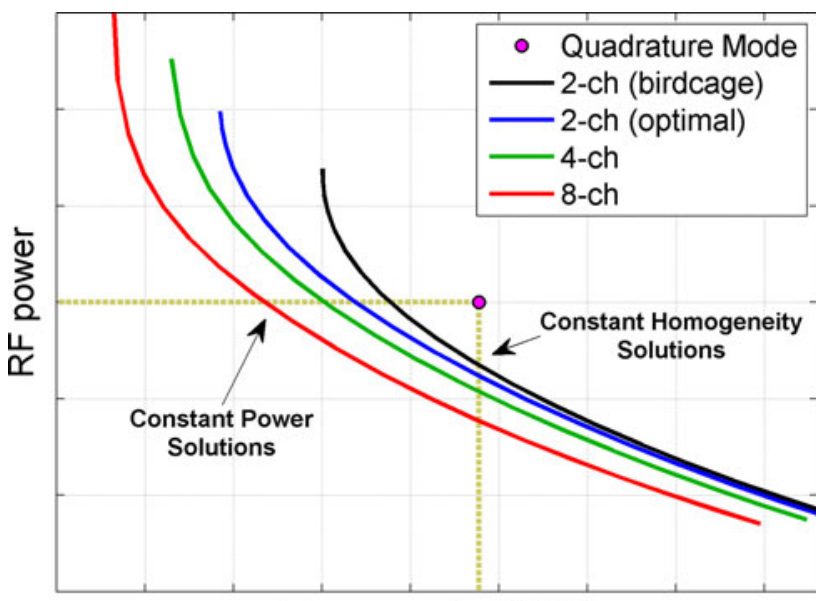

Inhomogeneity (RMSE)

Fig. 1 Trade off between relative RF power and inhomogeneity (RMSE) for different coil configurations. Each curve is generated by performing the RF shimming calculation for multiple values of regularization parameter $\lambda$. Solutions for constant RF power are indicated by the intersection of the horizontal dotted line with the other curves. Solutions for constant homogeneity are indicated by intersection with the vertical dotted line

repetition times of $30 \mathrm{~ms}$ and $150 \mathrm{~ms}$. A T1-weighted turbo spin echo (T1w-TSE) sequence $(440 \mathrm{~mm} \times 220 \mathrm{~mm}$ FOV, $400 \times 318$ matrix, TR $=605 \mathrm{~ms}, \mathrm{TE}=10 \mathrm{~ms}$, TSE turbo factor $=3$ ) and a T2-weighted turbo spin echo (T2w-TSE) sequence $(440 \mathrm{~mm} \times 220 \mathrm{~mm} \mathrm{FOV}, 400 \times 318$ matrix, $\mathrm{TR}=2,000 \mathrm{~ms}, \mathrm{TE}=100 \mathrm{~ms}$, TSE turbo factor $=10$ ) were chosen for body imaging so the effect of varying the number of channels had on image quality could be assessed. All imaging was performed in the transverse plane using a single slice acquisition. The TSE sequences outlined above are often used to acquire multiple interleaved slices; use of a single slice allows the SAR to be kept as low as possible while maintaining the same contrast parameters as used in clinical examinations. A full examination, consisting of $\mathrm{B}_{1}{ }^{+}$mapping, RF shim calculation and acquisition of images for all channel combinations, could be completed within $45 \mathrm{~min} . \mathrm{B}_{1}{ }^{+}$maps were also obtained for each optimized shim setting to allow comparison between the predicted and achieved shimmed $\mathrm{B}_{1}{ }^{+}$distributions, independent of the receiver coil.

RF safety and SAR analysis

Accurate calculation of SAR, particularly the local SAR, requires subject specific E-field models based on the individual's anatomy. Obtaining this information and completing the required calculations remains a challenge to achieve during an examination and is a current research topic in its own right $[14,15]$. In this study whole body SAR was dealt with simplistically using the $\|\mathbf{R b}\|^{2}$ term in the RF shimming cost function (Eq. 2). This method allowed for a time-efficient, indirect minimisation of global 
SAR through the limiting of total RF power. A singlesubject, single-position E-field model was also used to calculate the local SAR prior to every scan performed using the method from Ref. [16], allowing for quantification of the SAR reduction potential of multiple transmit channels. The E-field model is for a large male subject, positioned with torso at isocentre, therefore the results acquired are unlikely to be accurate for different body shapes and different body positions relative to the RF coils. Due to the uncertainty in the SAR taken from this model, a maximum estimated SAR level of $10 \%$ of the relevant regulatory limit [17] was allowed for any imaging sequence, to allow a tenfold margin for error.

$\mathrm{B}_{1}{ }^{+}$homogeneity and image quality assessment

Homogeneity was assessed for acquired $\mathrm{B}_{1}{ }^{+}$maps using both root mean square error (RMSE) and coefficient of variation $\left(C_{\mathrm{V}}\right)$. Both measures considered only the homogeneity of the magnitude of the measured $\mathrm{B}_{1}{ }^{+}$. RMSE quantifies the deviation in absolute terms from the desired field pattern, and is equivalent to the square root of the first term in Eq. 2. The coefficient of variation (standard deviation divided by mean) is purely a measure of spatial homogeneity; unlike RMSE, $C_{\mathrm{v}}$ is not altered by scaling the $\mathrm{B}_{1}{ }^{+}$fields. We hypothesised that increasing the number of channels would improve homogeneity, and therefore decrease the $C_{\mathrm{V}}$. Statistical analysis was carried out on the $C_{\mathrm{V}}$ data using SPSS (IBM). KolmogorovSmirnov tests were used to test the normality of the data (there was no evidence for non-normality), then paired sample t-tests were used to examine differences in $C_{\mathrm{V}}$ for incremental increases in the number of channels controlled ( 2 vs. 1 (quad), 4 vs. 2,8 vs. 4 ). The requirement for statistical significance was $p<0.05$ for one-tailed comparisons (i.e. the alternative hypothesis is that $C_{\mathrm{V}}$ for the configuration with more channels is less).

A blind grading system was used to assess changes in imaging performance. An experienced radiologist was given all images in a randomised order and asked to grade them according to a five-point scoring system, previously described by Willinek et al. [4], but modified as all volunteers were healthy and no pathology was assessed. Perceived image quality was rated on a scale of $1-5$, with 1 being an image of non-diagnostic quality and 5 demonstrating optimal contrast with no $\mathrm{B}_{1}{ }^{+}$inhomogeneity artefacts.

\section{Results}

$\mathrm{B}_{1}{ }^{+}$homogeneity and Image quality improvements

A consistent pattern of substantial inhomogeneity was found in the $\mathrm{B}_{1}{ }^{+}$field generated by quadrature excitation, in both the pelvis and thighs of all volunteers. The inhomogeneity was characterised by diagonally offset areas of low $\mathrm{B}_{1}{ }^{+}$intensity in the anterior and posterior regions. This resulted in areas of very low signal intensity in the resulting $\mathrm{T}_{1} \mathrm{w}$-TSE images; the $\mathrm{T}_{2} \mathrm{~W}$-TSE images were more robust to the inhomogeneity. The $\mathrm{B}_{1}{ }^{+}$fields and the corresponding $\mathrm{T}_{1} \mathrm{~W}$ and $\mathrm{T}_{2} \mathrm{~W}$ images acquired in quadrature mode for all volunteers are shown in Fig. 2 for the pelvis and Fig. 3 for the thighs. Note that the variability seen in the average $\mathrm{B}_{1}{ }^{+}$value between different subjects in Fig. 2 is the result of inaccuracy in the scanner's standard global poweroptimisation procedure [18].

Figure 4 contains RF homogeneity, power and SAR estimates for the pelvis and thigh experiments. All shim solutions had an approximately constant RF forward drive, relative to quadrature mode, as can be seen in parts e, $\mathrm{f}$. The maximum local SAR as indicated by the E-field model based SAR calculator is displayed on parts $g$, $h$; note that the local SAR measurements are quoted for the AFI $\mathrm{B}_{1}{ }^{+}$ mapping sequence. This figure shows that by increasing the number of transmit channels, the $\mathrm{B}_{1}{ }^{+}$field can be made progressively more homogeneous for both the pelvis and thighs. The results of paired sample $t$-tests comparing $C_{\mathrm{V}}$ for configurations with successively increasing numbers of channels showed that improvements were significant (Table 1) for both anatomies studied and for all increases in channel number. Examples of the effect of these improvements in single volunteers can be seen in Figs. 5 and 6 , which contain the $\mathrm{B}_{1}{ }^{+}$maps, T1w-TSE and T2wTSE images obtained for each configuration from a single volunteer.

The mean maximum local SAR measurements from the online SAR model decreased with increasing numbers of channels for the pelvis experiment, although 4- and 8-channel configurations performed similarly. In the thighs there was also a gradual reduction with the exception of the 2-channel (non-birdcage) configuration which achieved a much lower calculated average local SAR than the other configurations. When transmitting in single-channel (nominal quadrature) mode, the maximum local SAR for the AFI sequence is always $3 \mathrm{~W} / \mathrm{kg}$ according to the experimental online electric field model as no phase/ amplitude modifications have been made and the system is calibrated.

The blind grading results from the anatomical images for all coil configurations averaged over all subjects are displayed in Table 2. In the case of the T1w images, increasing channel numbers also increases perceived image performance for both anatomies tested. For pelvic imaging there were no perceived improvements in the $\mathrm{T} 2 \mathrm{w}$ images when increasing the number of transmit channels; however, a slight improvement was noted when imaging the thighs. 


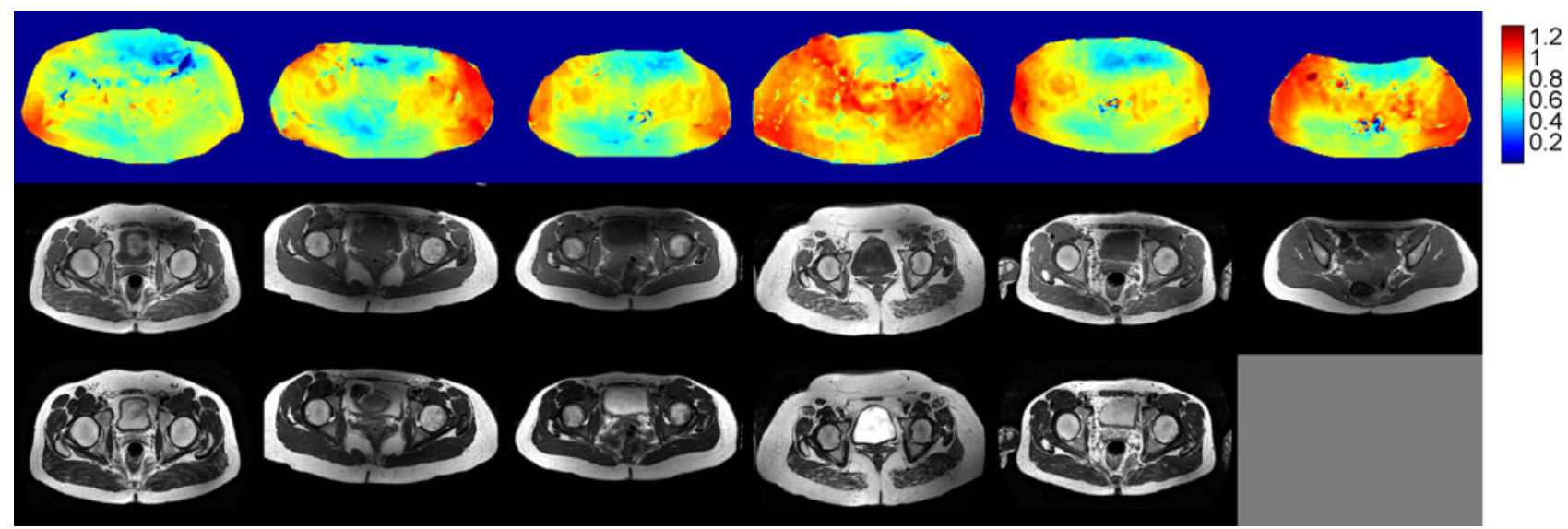

Fig. 2 Nominal quadrature mode axial pelvis results for multiple volunteers. Axial AFI transmit sensitivity maps $(\mathrm{S}(\mathbf{r}))$ (top), $\mathrm{T}_{1} \mathrm{w}$-TSE images (middle) and $\mathrm{T}_{2} \mathrm{~W}$-TSE images (bottom). Homogeneity is variable in all volunteers. Shading artefacts are observed particularly in the $\mathrm{T}_{1} \mathrm{w}$ images. Note that the $\mathrm{T} 2 \mathrm{w}$ image is missing for one volunteer because technical problems led to the examination being terminated before all data had been acquired

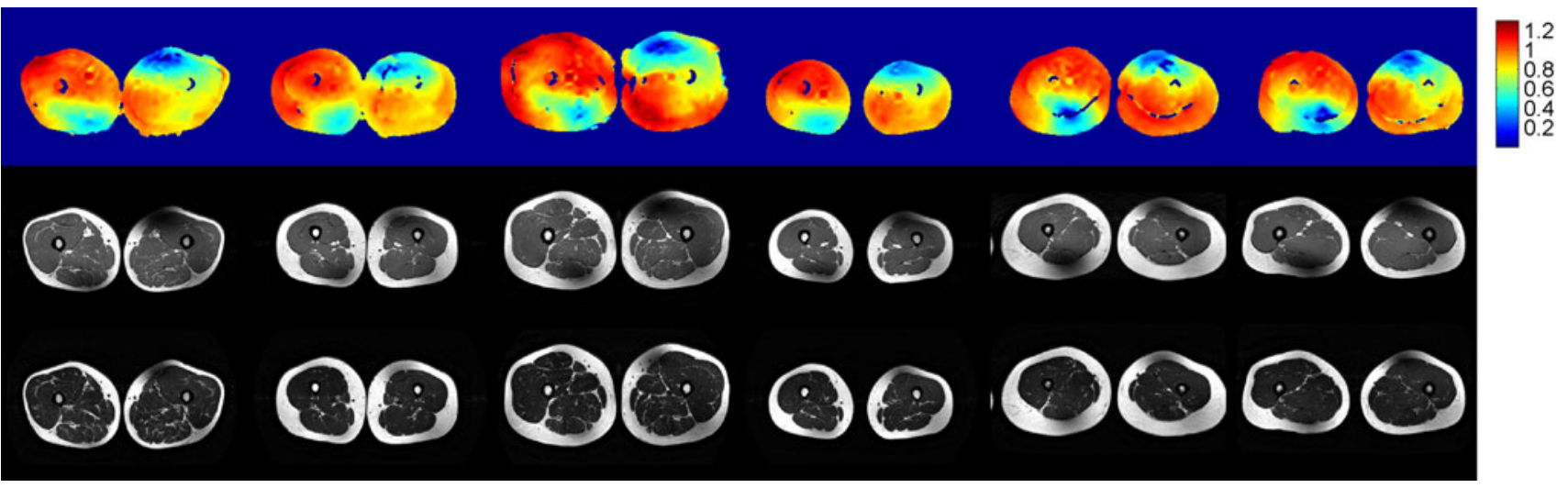

Fig. 3 Nominal quadrature mode axial thigh results for multiple volunteers. Axial AFI transmit sensitivity maps (top), $\mathrm{T}_{1} \mathrm{w}-\mathrm{TSE}$ images (middle) and $\mathrm{T}_{2} \mathrm{~W}$-TSE images (bottom). Significant areas of low sensitivity are seen in the upper right and lower left portions of the field of view with corresponding shading artefacts present on the $\mathrm{T}_{1} \mathrm{w}$ and $\mathrm{T}_{2} \mathrm{w}$ images

\section{Discussion}

In this study, conducted at $3 \mathrm{~T}$, pronounced areas of $\mathrm{B}_{1}^{+}$ inhomogeneity were found in the body with the areas of low $\mathrm{B}_{1}^{+}$intensity following a distinctive diagonal characteristic of 'elliptic' shaped loads, which the human body loosely resembles [19]. Our results show that increasing the number of RF channels, and hence the number of effective degrees of freedom, leads to successive improvement in the achievable $\mathrm{B}_{1}{ }^{+}$homogeneity. This is visible from the diagram in Fig. 1, which was generated using one of the datasets included in the study. The solutions found for configurations with more degrees of freedom are nested within those found with fewer degrees of freedom, indicating that a more favourable trade-off between power and inhomogeneity can be achieved by increasing the channel 
Fig. 4 Results for experiments designed to maximise homogeneity at constant RF power. Pelvis results are shown in the left hand column, with thigh results in the right column. The maximum whisker length on all box plots is $1.5 \times$ the interquartile range; all points outside this are deemed outliers and marked with a red cross. a, b RMSE inhomogeneity reductions achieved by increasing the number of channels. c, d C $\mathrm{C}_{\mathrm{V}}$ inhomogeneity versus channel configuration. Note that both $\operatorname{RMSE}(\mathrm{a} / \mathrm{b})$ and $\mathrm{C}_{\mathrm{V}}(\mathrm{c} / \mathrm{d})$ were measured from acquired $\mathrm{B}_{1}{ }^{+}$ maps, e, f RF power was held constant with respect to quadrature mode. $\mathbf{g}, \mathbf{h}$ Local SAR estimate from numerical model for AFI $\mathrm{B}_{1}{ }^{+}$mapping sequence versus channel configuration
Pelvis
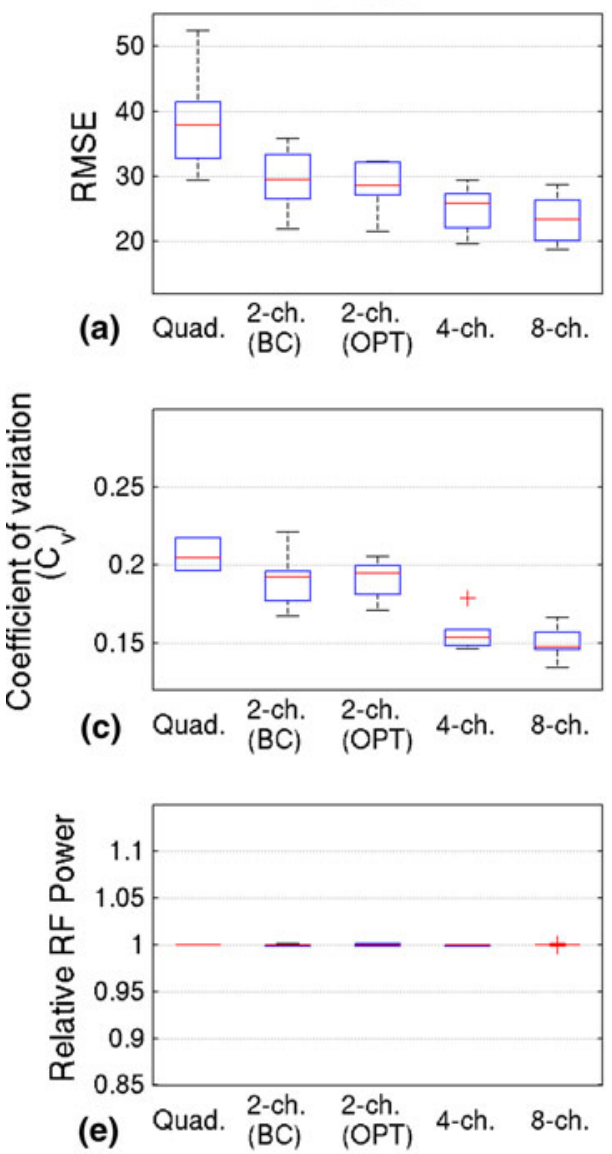

్ㅠㅁ (e)

(BC)

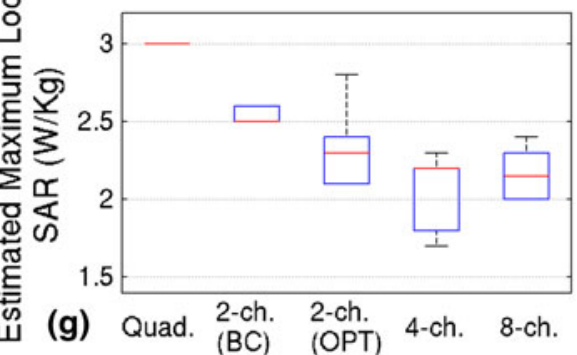

Thighs
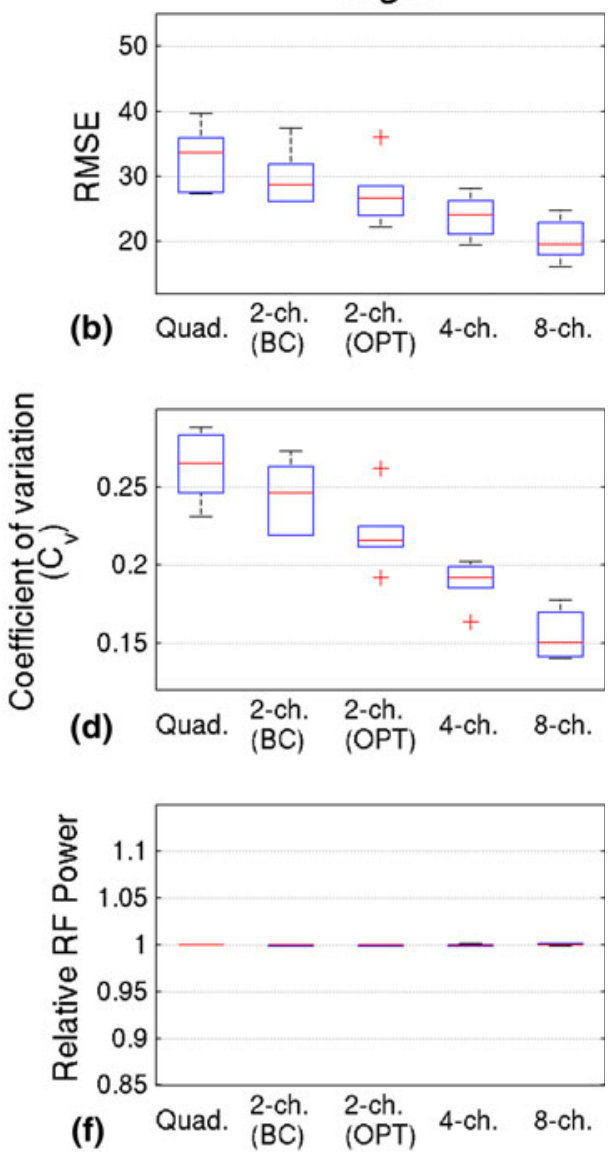

『్

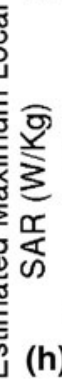

(h) Quad. 2-ch. 2-ch. 4-ch. 8-ch.

Table 1 Table of $p$ values for pairwise comparison of $C_{\mathrm{V}}$ measured from acquired $\mathrm{B}_{1}{ }^{+}$maps for configurations with increasing numbers of channels

\begin{tabular}{llll}
\hline Anatomy & $p$ values & & \\
\cline { 2 - 4 } & 2-channel (opt) compared with Quadrature & 4-channel compared with 2-channel (opt) & 8-channel compared with 4-channel \\
\hline Pelvis & 0.0026 & 0.0005 & 0.0075 \\
Thigh & 0.0038 & 0.0020 & 0.0004
\end{tabular}

Taking 0.05 as the threshold for significance, increasing from one to two, two to four channels and four to eight channels yielded significant improvements in homogeneity in all cases. Note that only the optimal 2-channel configuration (not the 'birdcage' configuration) is shown here to reduce the number of comparisons

number. A systematic study using multiple volunteers confirmed that increasing the number of channels from one to two, two to four, and four to eight all resulted in statistically significant improvements in homogeneity. Improved RF homogeneity also led to a quantifiable improvement in image quality as measured by a blinded 


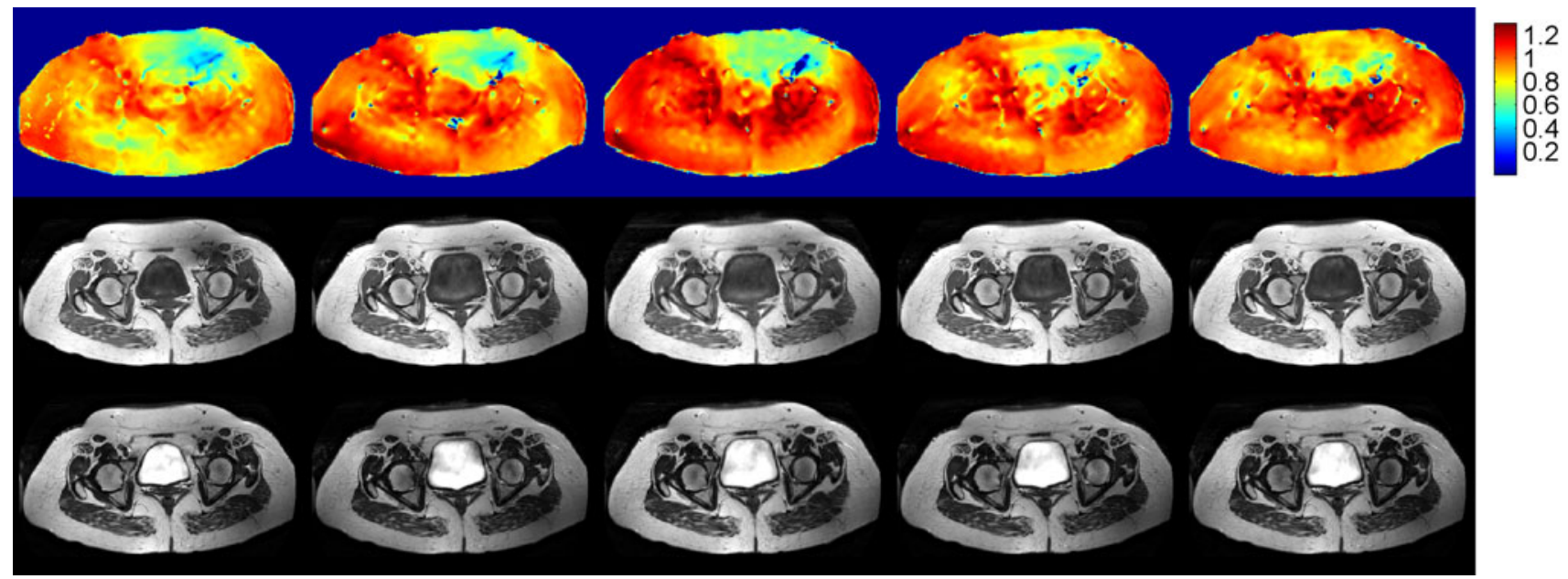

Fig. 5 Example data from pelvis scans of one volunteer. Top AFI axial pelvic $\mathrm{S}(\mathbf{r})$ maps, middle $\mathrm{T}_{1} \mathrm{w}-\mathrm{TSE}$ and bottom $\mathrm{T}_{2} \mathrm{~W}$-TSE axial pelvic images; columns from left to right: quadrature, 2-channel (birdcage), 2-channel (optimal), 4-channel and 8-channel. The $\mathrm{T}_{2} \mathrm{~W}$ images appear more robust to $\mathrm{B}_{1}{ }^{+}$inhomogeneity than the $\mathrm{T} 1 \mathrm{w}$ sequence

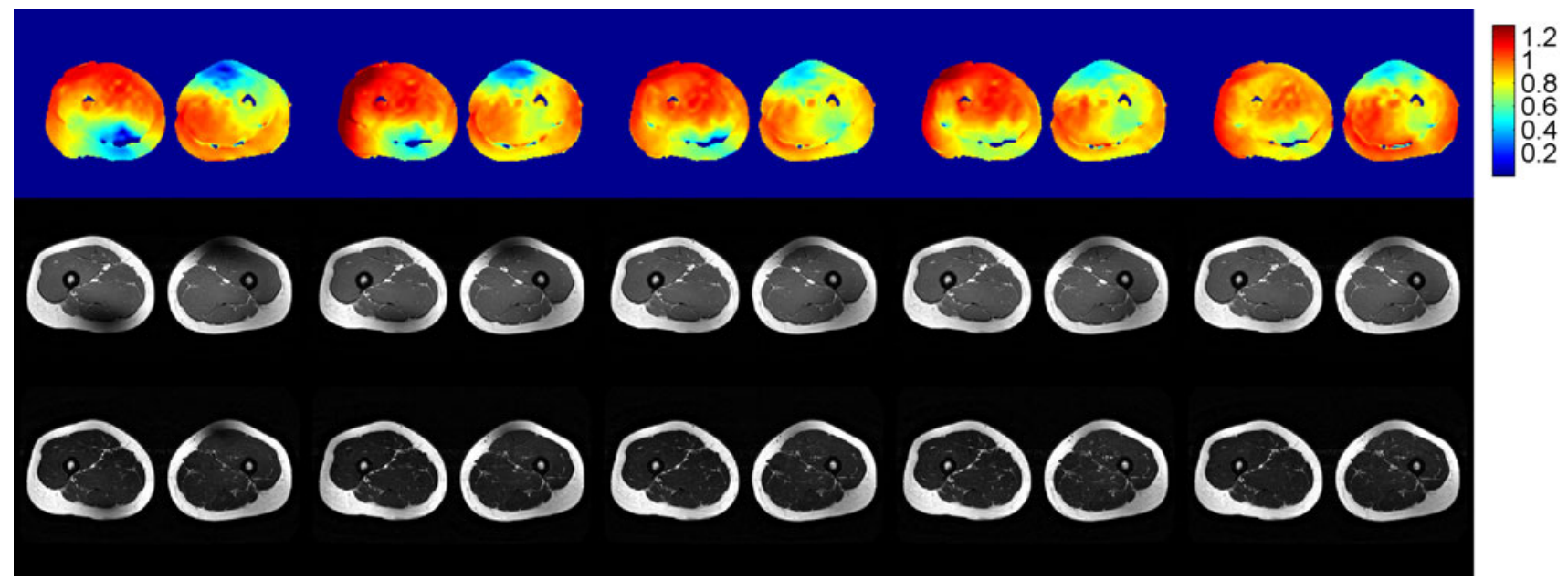

Fig. 6 Example data from thigh scans of one volunteer. Top AFI axial thigh $\mathrm{S}(\mathbf{r})$ maps, middle $\mathrm{T}_{1} \mathrm{w}-\mathrm{TSE}$ and bottom $\mathrm{T}_{2} \mathrm{~W}$-TSE axial pelvic images; columns from left to right: quadrature, 2-channel (birdcage), 2-channel (optimal), 4-channel and 8-channel. The $\mathrm{T}_{2} \mathrm{~W}$ images appear more robust to $\mathrm{B}_{1}^{+}$inhomogeneity than the $\mathrm{T} 1 \mathrm{w}$ sequence

Table 2 Results of blinded image quality grading (mean \pm SD across all subjects) using five point scale adapted from Willinek et al. [4]

\begin{tabular}{|c|c|c|c|c|c|c|}
\hline Anatomy & Imaging sequence & Quadrature & 2-channel (birdcage) & 2-channel (optimal) & 4-channel & 8-channel \\
\hline \multirow[t]{2}{*}{ Pelvis } & $\mathrm{T} 1 \mathrm{w}$ & $4.00 \pm 0.63$ & $4.33 \pm 0.52$ & $4.33 \pm 0.52$ & $4.50 \pm 0.55$ & $4.67 \pm 0.52$ \\
\hline & $\mathrm{T} 2 \mathrm{w}$ & $4.20 \pm 0.45$ & $4.60 \pm 0.55$ & $4.60 \pm 0.55$ & $4.60 \pm 0.55$ & $4.60 \pm 0.55$ \\
\hline \multirow[t]{2}{*}{ Thigh } & $\mathrm{T} 1 \mathrm{w}$ & $2.50 \pm 0.55$ & $3.20 \pm 0.84$ & $3.67 \pm 1.03$ & $4.33 \pm 0.52$ & $4.67 \pm 0.52$ \\
\hline & $\mathrm{T} 2 \mathrm{w}$ & $4.00 \pm 0.63$ & $4.60 \pm 0.55$ & $4.60 \pm 0.55$ & $4.83 \pm 0.41$ & $4.83 \pm 0.41$ \\
\hline
\end{tabular}

evaluation, especially in $\mathrm{T} 1$ weighted images. The T2 weighted TSE sequence used was found to be more robust to $\mathrm{B}_{1}{ }^{+}$homogeneity. The amplitude of a single spin echo produced by refocusing flip angle $\alpha$ is proportional to $\sin ^{2}(\alpha / 2)$, whereas the pseudo steady-state echo amplitude produced by a TSE sequence with multiple refocusing is 
Fig. 7 Comparisons of homogeneity, power requirements and local SAR with different numbers of channels when performing RF shimming at a fixed level of homogeneity. Unlike Fig. 4, homogeneity values $\left(\mathrm{RMSE} / \mathrm{C}_{\mathrm{V}}\right)$ are predicted rather than measured. Pelvis results are shown in the left column, thigh results on the right. a, b RMSE is held the same for all configurations; variability comes from inter-subject differences. $\mathbf{c}, \mathbf{d ~ C}_{\mathrm{v}}$ falls with increasing channel count, indicating that although the RMSE is held constant the actual field patterns do vary. e, f RF power requirements fall as the number of channels increases. $\mathbf{g}$, h Local SAR prediction of AFI $\mathrm{B}_{1}^{+}$mapping sequence acquired with power reduction shims
Pelvis
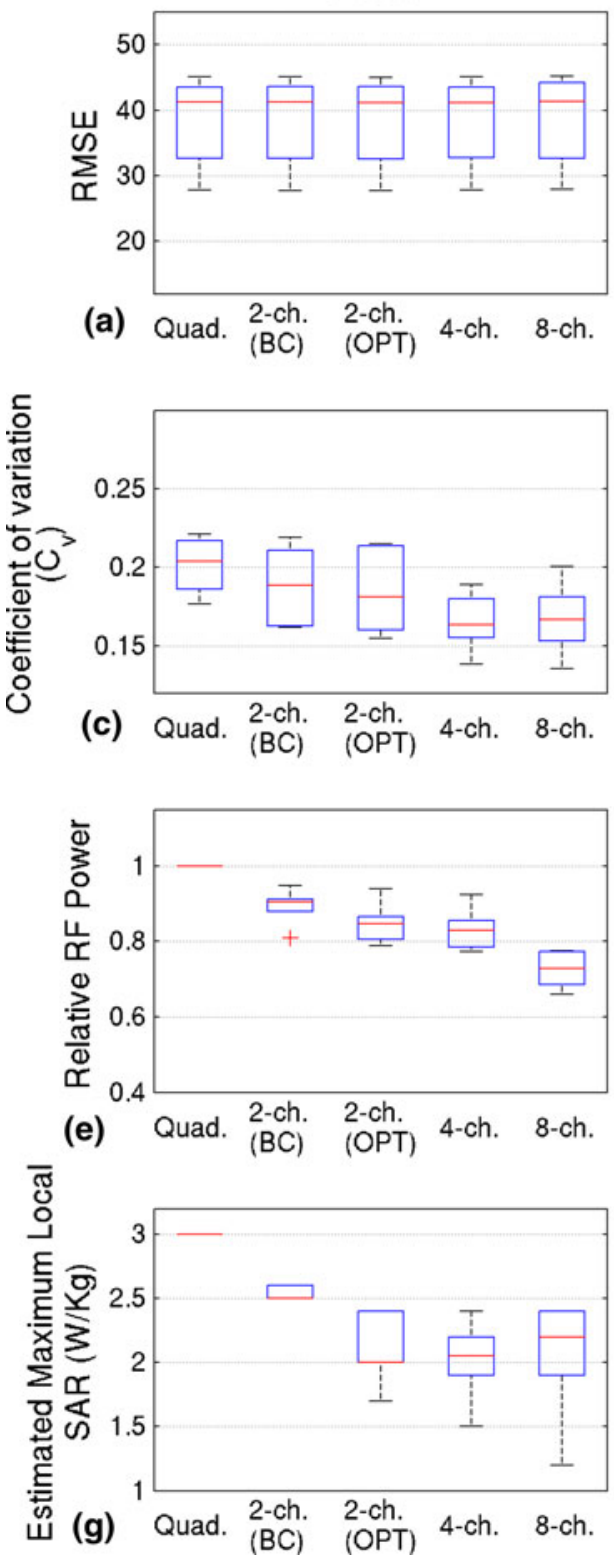

Thighs
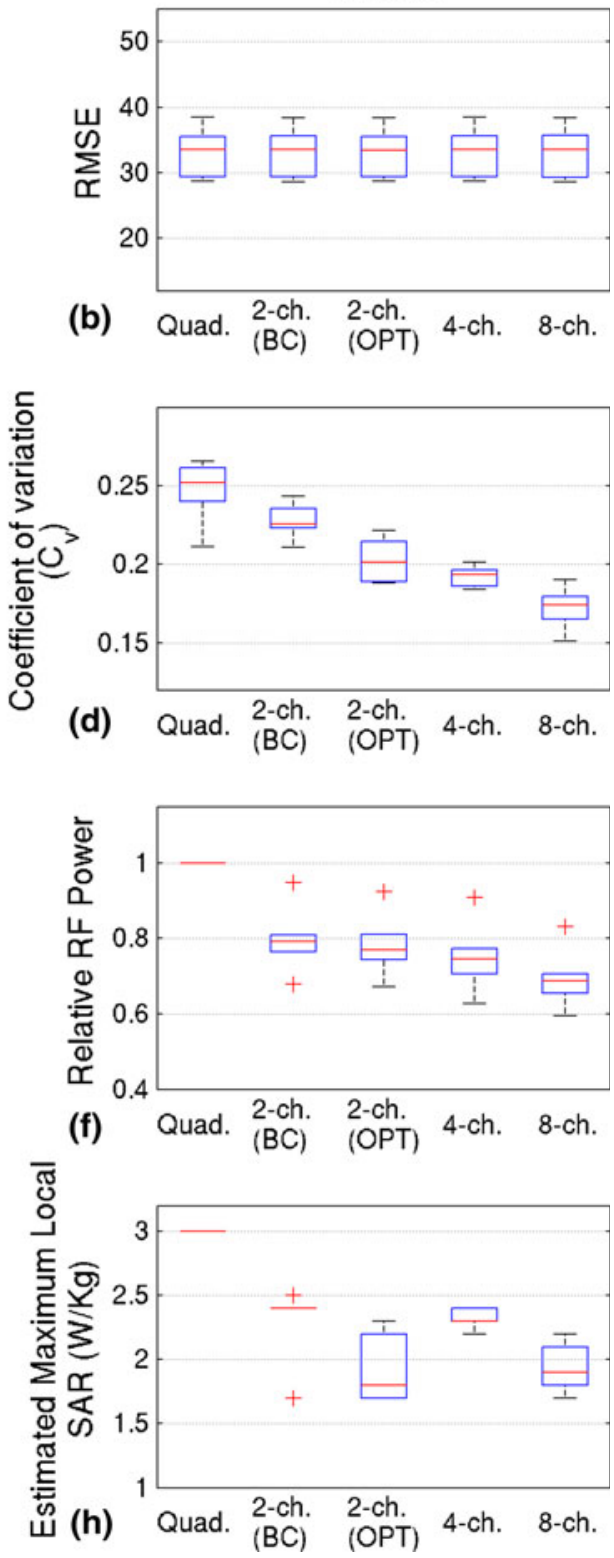

proportional to $\sin (\alpha / 2)$ [20]. For both the T1 and T2 weighted sequences, $\alpha$ was $130^{\circ}$, however the former used centric phase encoding, while the latter used linear. As a result it would be expected that the $\mathrm{T} 1$ weighted images would be more influenced by the first echo (proportional to $\sin ^{2}(\alpha / 2)$ ) while the T2 images would be closer to the pseudo steady-state $(\sin (\alpha / 2))$, which is in accordance with the finding that the T2 weighted sequence is more robust to variations in $\mathrm{B}_{1}^{+}$.

As well as $\mathrm{B}_{1}{ }^{+}$field homogeneity, parallel transmission also allows greater control over both global and local SAR. RF shims were calculated for maximal homogeneity at a fixed RF drive power level, where drive power is the sum of squares of the RF drive levels supplied to each channel, quantified by $\|\mathbf{R} \mathbf{b}\|^{2}$. A single station E-field model for a single subject was available for the estimation of peak local SAR for these solutions (Fig. $4 \mathrm{~g}$, $\mathrm{h}$ for pelvis and thigh respectively). These results indicate that there was some reduction in local SAR for the shimmed solutions, but importantly none of the shimmed solutions produced a higher local SAR than quadrature mode, according to the model. It should be noted that this optimisation did not aim to minimise local SAR, and in fact held relative power constant. A second set of experiments which held homogeneity constant and aimed to reduce total power did indeed show consecutive reductions in total power as channel number increased. The local SAR, as predicted by the model, also decreased when compared with quadrature 
mode, but again decreases were erratic. This is partly to be expected because the SAR model did not accurately describe the imaging scenarios, either in the positioning or the size and build of the subject. Personalised models for each subject might allow a subject-specific estimation of the local SAR within the current framework. These could be used to much greater effect by allowing the replacement of the regularization term in Eq. 2 with a term based directly on a true SAR estimate. A recent study of this type by Homann et al. [15] investigated shimming with an 8-channel coil of a similar design to the one used in this study. In that study, it was found that including a subjectspecific SAR model when determining shim settings produced substantial SAR reductions, which is in line with our findings using a single standard model and in relation to total input power. The method employed in the current study, in which an L-curve is calculated to explore balancing $\mathrm{B}_{1}{ }^{+}$homogeneity with total drive power, or ideally with peak local SAR, allows flexible choice of operating point. In this study, constant power or constant homogeneity solutions were examined, but in practice any point along the curves could be selected, depending on the situation. As indicated by the imaging examples presented in Figs. 2, 3, 5 and 6, different sequences have variable sensitivity to $\mathrm{B}_{1}^{+}$inhomogeneity. The T2w sequence employed here is more robust, and so a lower power solution might be selected to increase efficiency, whereas other parts of an examination may be much more vulnerable and so warrant a different homogeneity-power tradeoff. This allows flexible balancing of SAR load across a whole examination. At the other extreme, Fig. 1 shows that it is possible to achieve highly homogeneous $\mathrm{B}_{1}{ }^{+}$fields over a large (axial) field of view with a multi-transmit system at 3T if the total power is not a concern. However, useful solutions emerge as soon as any power considerations are introduced, in the case of the method used here, by setting $\lambda$ to any non-zero value.

The lack of suitability of the SAR model for the subjects imaged meant that the true SAR values were uncertain. For this reason, a conservative approach was taken, and imaging was limited to run at $10 \%$ of the relevant SAR limit. This limitation is the subject of a considerable research effort in the wider community: the use of generic models [21], simplified models generated in real time [22] or even actual in situ measurement of the SAR distribution [23] are all current possibilities. More accurate models would allow subject-specific SAR reductions to be calculated; if predictions could be treated with confidence, then reductions in quoted SAR would be translatable into an increase in permissible RF duty cycle, which can be used to reduce $\mathrm{TR}$ or increase the maximum number of slices within a fixed TR. If the number of slices were increased, extra $\mathrm{B}_{1}{ }^{+}$maps would also need to be acquired, potentially slowing the acquisition process. New methods such as that in Ref. [24] provide the possibility of rapid $\mathrm{B}_{1}{ }^{+}$measurement over large volumes, alleviating this issue. Similarly, while computation times were acceptable for this study, they would not scale well to multiple slices. The code used was, however, not optimized for speed since it was not an issue here, though it could be accelerated if necessary.

The human body, with its approximately elliptic cylindrical geometry, produces field variation mainly in the axial plane [19]. As a result, prevailing coil designs consist of elements distributed azimuthally around the magnet, giving maximal axial control. Consequently, in this study all imaging and field measurements were obtained in axial planes, where the effects of field inhomogeneity are generally greatest and the existing coil design offers its maximum flexibility in field control. We have found qualitatively similar performance differences between the different channel combinations for coronal orientation scanning. Given the asymmetry in the coil design, with its lack of any head-foot segmentation, the manner in which changing the orientation of the scanning plane modulates the magnitude of the effect of the number of degrees of freedom exploited is likely to be strongly influenced by the properties of the coil. A systematic investigation of these effects in the context of an array configuration that has multiple elements arrayed in all three directions around the body will be of further interest and the current work suggests that such additional degrees of freedom would result in a powerful capacity for homogeneity control. Similarly, while this study was conducted purely at $3 \mathrm{~T}, \mathrm{~B}_{1}{ }^{+}$inhomogeneity issues are greater at higher field strengths. The results of this work cannot directly be translated to these other scenarios, but they do indicate that transmit arrays, perhaps with more than 8 channels, could mitigate related issues at field strengths greater than 3T.

\section{Conclusion}

The impact the number of RF transmit channels has on $\mathrm{B}_{1}{ }^{+}$ homogeneity, RF power requirements/SAR reduction and image quality when performing subject-specific RF shimming was investigated. Increasing the number of transmit channels was found to yield progressive improvements in $\mathrm{B}_{1}{ }^{+}$homogeneity achieved under approximately constant total drive power, or reduction in total drive required to achieve the same level of $\mathrm{B}_{1}{ }^{+}$homogeneity. The regularised shimming approach adopted in this study allows $\mathrm{B}_{1}{ }^{+}$ homogeneity and total RF power to be traded against one another and the balance adjusted throughout an examination to optimise imaging performance and control RF power deposition. RF shimming with large coil arrays allows for significant increases in homogeneity, beyond 
those found with 2-channels. In this study, the maximum number of channels was 8 . Potentially, further performance increases could be achieved in some anatomies with a greater number of transmit channels, with the use of suitable array coils.

Acknowledgments The authors thank Philips Healthcare for technical support during this study and in particular Dr Paul Harvey for useful discussions. This work was partly supported by the EPSRC (Grant Number: EP/H046410/1).

Open Access This article is distributed under the terms of the Creative Commons Attribution License which permits any use, distribution, and reproduction in any medium, provided the original author(s) and the source are credited.

\section{References}

1. Ibrahim TS, Lee R, Baertlein BA, Abduljalil AM, Zhu H, Robitaille P-ML (2001) Effect of RF coil excitation on field inhomogeneity at ultra high fields: a field optimized TEM resonator. Magn Reson Imaging 19(10):1339-1347

2. Setsompop K, Alagappan V, Gagoski B, Witzel T, Polimeni J, Potthast A, Hebrank F, Fontius U, Schmitt F, Wald LL, Adalsteinsson E (2008) Slice-selective RF pulses for in vivo B1+ inhomogeneity mitigation at 7 tesla using parallel RF excitation with a 16-element coil. Magn Reson Med 60(6):1422-1432

3. Vernickel P, Roschmann P, Findeklee C, Ludeke KM, Leussler C, Overweg J, Katscher U, Grasslin I, Schunemann K (2007) Eight-channel transmit/receive body MRI coil at 3T. Magn Reson Med 58(2):381-389

4. Willinek WA, Gieseke J, Kukuk GM, Nelles M, Konig R, Morakkabati-Spitz N, Traber F, Thomas D, Kuhl CK, Schild HH (2010) Dual-source parallel radiofrequency excitation body MR imaging compared with standard MR imaging at $3.0 \mathrm{~T}$ : initial clinical experience 1. Radiology 256(3):966-975

5. Kukuk GM, Gieseke J, Weber S, Hadizadeh DR, Nelles M, Traber F, Schild HH, Willinek WA (2011) Focal liver lesions at 3.0 T: lesion detectability and image quality with $\mathrm{T} 2$-weighted imaging by using conventional and dual-source parallel radiofrequency transmission. Radiology 259(2):421-428

6. Kassakian P (2006) Convex approximation and optimization with applications in magnitude filter design and radiation pattern synthesis. Thesis. University of California at Berkeley

7. Setsompop K, Wald LL, Alagappan V, Gagoski BA, Adalsteinsson E (2008) Magnitude least squares optimization for parallel radio frequency excitation design demonstrated at 7 Tesla with eight channels. Magn Reson Med 59(4):908-915

8. Yarnykh VL (2007) Actual flip-angle imaging in the pulsed steady state: a method for rapid three-dimensional mapping of the transmitted radiofrequency field. Magn Reson Med 57(1): 192-200

9. Nehrke K (2009) On the steady-state properties of actual flip angle imaging (AFI). Magn Reson Med 61(1):84-92

10. Malik SJ, Kenny GD, Hajnal JV (2011) Slice profile correction for transmit sensitivity mapping using actual flip angle imaging. Magn Reson Med 65(5):1393-1399

11. Brunner DO, Pruessmann KP (2009) B1+ interferometry for the calibration of RF transmitter arrays. Magn Reson Med 61(6): $1480-1488$

12. Malik SJ, Larkman DJ, Hajnal JV (2009) Optimal linear combinations of array elements for B1 mapping. Magn Reson Med 62(4):902-909

13. Nehrke K, Boenert P (2010) Eigenmode analysis of transmit coil array for tailored B1 mapping. Magn Reson Med 63(3):754-764

14. Eichfelder G, Gebhardt M (2011) Local specific absorption rate control for parallel transmission by virtual observation points. Magn Reson Med 66(5):1468-1476

15. Homann H, Graesslin I, Eggers H, Nehrke K, Vernickel P, Katscher U, Dossel O, Boernert P (2011) Local SAR management by RF Shimming: a simulation study with multiple human body models. Magn Reson Mater Phy 25(3):193-204

16. Graesslin I, Homann H, Biederer S, Börnert P, Nehrke K, Vernickel P, Mens G, Harvey P, Katscher U (2012) A specific absorption rate prediction concept for parallel transmission MR. Magn Reson Med. doi:10.1002/mrm.24138

17. International Electro-technical Commission (IEC) (2008) Medical electrical equipment-Part 2-33: particular requirements for the safety of magnetic resonance equipment for medical diagnosis. IEC 60601-2-33 ed 2.2. Consolidated with amendments 1 and 2. IEC, Geneva

18. Carlson JW, Kramer DM (1990) Rapid radiofrequency calibration in MRI. Magn Reson Med 15(3):438-445

19. Sled JG, Pike GB (1998) Standing-wave and RF penetration artifacts caused by elliptic geometry: an electrodynamic analysis of MRI. Med Imaging IEEE Trans Med Imaging 17(4):653-662

20. Hennig J (1991) Echoes-how to generate, recognize, use or avoid them in MR-imaging sequences. Concept Magn Reson 3(3): 125-143

21. de Greef M, Ipek O, Raaijmakers AJE, Crezee J, van den Berg CAT (2012) Specific absorption rate intersubject variability in 7T parallel transmit MRI of the head. Magn Reson Med. doi: $10.1002 / \mathrm{mrm} .24378$

22. Homann H, Börnert P, Eggers H, Nehrke K, Dössel O, Graesslin I (2011) Toward individualized SAR models and in vivo validation. Magn Reson Med 66(6):1767-1776

23. Voigt T, Homann H, Katscher U, Doessel O (2011) Patientindividual local SAR determination: In vivo measurements and numerical validation. Magn Reson Med. doi:10.1002/mrm.23322

24. Nehrke K, Börnert P (2012) DREAM-a novel approach for robust, ultrafast, multislice B1 mapping. Magn Reson Med. doi: $10.1002 / \mathrm{mrm} .24158$ 